\title{
Proceedings of the Second NCI-SNMMI Workshop on Targeted Radionuclide Therapy
}

Frederic Fahey ${ }^{1}$, Katherine Zukotynski² ${ }^{2}$ Hossein $\mathrm{Jadvar}^{3}$, and Jacek Capala ${ }^{4}$, with input from the organizing committee, contributors, and participants of the second NCI-SNMMI Workshop on Targeted Radionuclide Therapy

\begin{abstract}
${ }^{I}$ Boston Children's Hospital and Harvard Medical School, Boston, Massachusetts; ${ }^{2}$ Sunnybrook Health Sciences Centre, University of Toronto, Toronto, Ontario, Canada, and Brigham and Women's Hospital, Harvard Medical School, Boston, Massachusetts;

${ }^{3}$ University of Southern California, Los Angeles, California; and ${ }^{4}$ National Cancer Institute, National Institutes of Health, Bethesda, Maryland
\end{abstract}

I 2013, the Society of Nuclear Medicine and Molecular Imaging (SNMMI) and the National Cancer Institute (NCI) partnered for the first time to host a joint workshop on targeted radionuclide therapy (TRT) (1). Broad discussion at that gathering suggested the need for a follow-up workshop bringing together both industry and government agencies in dialogue on educational and regulatory issues emphasizing further development of TRT. The second workshop, in October 2014, included individuals from multiple scientific disciplines, industry, government agencies, and international collaborators. The goal was to review what has been learned to date about the implementation of TRT, discuss the most promising agents moving forward, and investigate a path to bring them to the clinic. Cochaired by Drs. Frederic Fahey and Katherine Zukotynski, the 2-day event assembled a small but diverse group of stakeholders (Fig. 1) for discussion in both structured and open-forum formats. This white paper briefly summarizes the discussion on TRT that took place at the workshop and offers next-step recommendations.

\section{LESSONS LEARNED ABOUT TRT}

Through a series of presentations, individuals representing different professional perspectives on TRT offered insight on lessons learned and current challenges.

\section{Radiochemistry}

Dr. Cathy Cutler (University of Missouri) reviewed the status of TRT radiochemistry, described the experience at the University of Missouri Research Reactor Center, and explored the potential role of large-animal models in clinical trials of TRT.

Today, therapeutic use of radionuclides represents about $5 \%$ of nuclear medicine procedures. The optimal radionuclide for TRT is often target- and vector-dependent. Consideration should be given to decay characteristics (mode of decay, half-life, purity, specific activity), radiolabeling chemistry (simplicity, stability, pharmacokinetics), cost, and ability to access sufficient quantities for

Received Apr. 8, 2015; revision accepted May 11, 2015.

For correspondence or reprints contact: Frederic Fahey, Boston Children's Hospital, 300 Longwood Ave., Boston, MA 02115.

E-mail: frederic.fahey@childrens.harvard.edu.

Published online May 21, 2015.

COPYRIGHT (c) 2015 by the Society of Nuclear Medicine and Molecular Imaging, Inc.

DOI: $10.2967 /$ jnumed.115.159038 clinical trials or routine use. The choice of chelate, method of radiolabeling, time for production and formulation, ability to automate, and methods of purification and quality control are all important. The mode of production can affect sample purity and cost, and the ability to automate manufacturing can affect availability.

The advantages of TRT include the ability to tailor tumor dose versus normal-tissue dose (which is not possible with chemotherapy), selective targeting of disease, and cross-fire irradiation, among others. Cross-fire irradiation allows killing of tumor cells that are not directly targeted, which is important for treating heterogeneous tumor tissue.

Traditionally the Food and Drug Administration (FDA) has mandated large-batch radiopharmaceutical production to ensure uniformity and enable extensive quality control testing. However, few sites have the necessary facilities or expertise. An alternative production method was implemented for ${ }^{131}$ I-tositumomab (Bexxar; GlaxoSmithKline) and ${ }^{90}$ Y-ibritumomab tiuxetan (Zevalin; Spectrum Pharmaceuticals, Inc.); kits containing the antibody and radionuclide were shipped to on-site radiopharmacies, which produced the compound as needed. Although this method eliminated large-scale centralized production, access to kits and specialized transportation were problematic. Another alternative was the development of generators. However, access to generators was limited. Finally, the production of medium-sized radiopharmaceutical batches at a limited number of regional centers, similar to the method for manufacturing PET radiopharmaceuticals, has been suggested. This model may be more cost-effective than large-batch production, eliminate reliance on a single site, and enable hospitals and clinics to order a unit dose for administration, which is often preferred.

The University of Missouri Research Reactor Center experience supporting the ${ }^{166} \mathrm{Ho}-1,4,7,10$-tetraazacyclododecane-1,4,7,10tetramethylene-phosphonate (DOTMP) clinical trial illustrated challenges associated with radiopharmaceutical production and distribution. The University of Missouri Research Reactor had to process $740-7,400 \mathrm{GBq}(20-200 \mathrm{Ci})$ of ${ }^{166} \mathrm{Ho}$, produce clinicalgrade ${ }^{166} \mathrm{Ho}$-DOTMP, and access a high-flux region $2-4 \mathrm{~d}$ per week for insertion and removal of targets. Dedicated hot cells and a 6-barrel flux trap with tubes that could be removed while the reactor was running were used. A partnership with industry was forged to bolster cGMP experience.

The experience at the University of Missouri with a canine model for testing radioassays illustrated the potential role of 


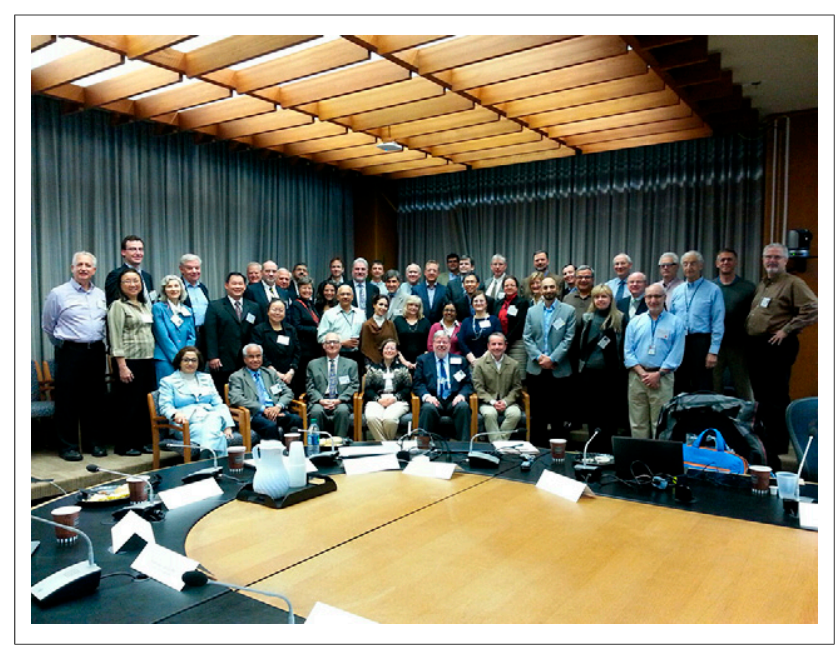

FIGURE 1. Participants of the second NCl-SNMMI Workshop on Targeted Radionuclide Therapy.

large-animal models in TRT clinical trials. To evaluate ${ }^{177} \mathrm{Lu}$-DOTMP toxicity, researchers from the University of Missouri studied the effects of radiopharmaceutical administration on dogs (2). The preliminary findings supported evaluation of the radiopharmaceutical as a potential therapy for primary and metastatic bone cancer in both dogs and humans. Trials in dog models of spontaneously occurring cancers that mimic the human conditions facilitate protocol optimization and translation to humans.

Dr. Cutler concluded her talk by addressing the role of radiobiology in TRT, an important and largely unexplored topic. She suggested that radiochemists have a key role to play in the future of TRT and that collaboration with both physicians and members of the public is needed to demonstrate the success and safety of TRT.

\section{Industry}

Dr. Lee Allen and Dr. Rick Satitpunwaycha (Spectrum Pharmaceuticals) provided insight into TRT commercialization challenges, focusing on ${ }^{90} \mathrm{Y}$-ibritumomab tiuxetan, a CD20-directed regimen available in more than 40 countries and the first radioimmunotherapy to receive FDA approval in the United States.

${ }^{90}$ Y-ibritumomab tiuxetan may be used to treat relapsed or refractory low-grade, follicular, or transformed B-cell non-Hodgkin lymphoma, including rituximab-refractory follicular non-Hodgkin lymphoma. In describing 2 key studies for FDA approval, Dr. Allen suggested that neither radioimmunotherapy response nor toxicity was an obstacle. The overall response rate of ${ }^{90}$ Y-ibritumomab tiuxetan was $74 \%$ in one study, a single-arm trial of 54 patients with relapsed, rituximab-refractory follicular lymphoma. The overall response rate was significantly higher in a second study, a randomized, open-label multicenter comparison with rituximab in 130 patients with relapsed or refractory low-grade or follicular nonHodgkin lymphoma ( $83 \%$ vs. $55 \%, P<0.001)$. The complete response rates to radioimmunotherapy were also high: $15 \%$ in one study and $38 \%$ in the other study. Regarding adverse effects, the principal toxicity was prolonged, severe cytopenia. The most common nonhematologic adverse reactions were fatigue (33\%), nasopharyngitis (19\%), and nausea (18\%). In a phase III randomized, controlled trial of ${ }^{90}$ Y-ibritumomab tiuxetan for consolidation in patients with advanced follicular non-Hodgkin lymphoma, the overall incidence of secondary malignancies was $13 \%$ (3). Dr. Allen noted that although oncologists are concerned about the potential for secondary malignancies, ${ }^{90} \mathrm{Y}$-ibritumomab tiuxetan toxicities are manageable, with the advantage of a one-time administration versus multiple cycles of chemotherapy.

However, despite the apparent effectiveness, safety, and convenience of radioimmunotherapy, use is low. ${ }^{90}$ Y-ibritumomab tiuxetan was used to treat 889 patients in 2010, compared with 551 in 2013, and was projected to be given to approximately 400 patients in 2014 . In an effort to augment data, the manufacturer recently initiated 3 randomized clinical trials including ${ }^{90}$ Y-ibritumomab tiuxetan, all of which have now been terminated because of low patient accrual. For example, the target accrual for a trial of patients with diffuse large B-cell lymphoma was 500, but after 2 y only 70 patients had been enrolled. Indeed, Dr. Satitpunwaycha said GlaxoSmithKline's decision to withdraw ${ }^{131}$ I-tositumomab from the market in February 2014 was because "use of the regimen has been extremely limited and is projected to decline." Only 55 doses of ${ }^{131}$ I-tositumomab were sold in North America in 2013.

Recently Spectrum Pharmaceuticals conducted a survey of oncologists that found several possible reasons for low ${ }^{90} \mathrm{Y}$-ibritumomab tiuxetan use: patient concern about radiation, physician concern about myelodysplastic syndrome, availability of new oral agents, the requirement for platelets to be over 100,000 and bone marrow involvement to be less than $25 \%$, and perception that ${ }^{131}$ I-tositumomab and ${ }^{90}$ Y-ibritumomab tiuxetan had been taken off the market.

Dr. Allen and Dr. Satitpunwaycha concluded that several issues need to be addressed for radioimmunotherapy to be adopted into clinical practice, including improving collaboration between physicians to allow easy access to radioimmunotherapy, training physicians to use radioimmunotherapy, bolstering support from opinion leaders, improving ease of assessing patient eligibility and posttherapy follow-up, and resolving reimbursement concerns with the Affordable Care Act. In short, ${ }^{90}$ Y-ibritumomab tiuxetan may be an effective therapy that is being avoided, at least in part, because of logistic challenges related to access, lack of experience, and competition from newer agents.

\section{Lymphoma Therapy}

Dr. Janis O'Malley (University of Alabama at Birmingham) discussed the role of radioimmunotherapy in lymphoma, using the experience with ${ }^{131}$ I-tositumomab and ${ }^{90}$ Y-ibritumomab tiuxetan as examples of why effective therapies sometimes fail after reaching the commercial market. Background was provided on the incidence of lymphoma in the United States, prognosis, and management (4-6).

Several trials have shown increased survival in patients with lymphoma treated with the anti-CD20 monoclonal antibody $(\mathrm{mAb})$ rituximab, which induces complement-dependent cytotoxicity and antibody-dependent cellular toxicity (7). Other immunotherapy agents approved for lymphoma include the anti-CD52 mAbs alemtuzumab and ofatumumab and the anti-CD30 antibodydrug conjugate brentuximab vedotin (8). Radioimmunotherapy with mAbs that bind the CD20 and CD30 antigen may be effective for patients with lymphoma. In the phase III FIT trial for patients with previously untreated, advanced follicular lymphoma, median progression-free survival (PFS) was 37 mo in the ${ }^{90}$ Y-ibritumomab tiuxetan arm, compared with 13.5 mo in controls $(P<0.001)(9)$. However, despite their success and the potential for using radioimmunotherapy to target antibody markers beyond CD20 and CD30, Dr. O'Malley noted that these agents have fallen 
out of favor with many oncologists. This may be due, at least in part, to concerns about cost, radiation effects, and a perceived lack of data from large randomized trials comparing radioimmunotherapy with nonradioactive lymphoma therapy. There is also concern about a potential negative financial effect from patient referral to a nuclear medicine physician or a radiation oncologist (10).

Dr. O'Malley reviewed the experience with ${ }^{131}$ I-tositumomab since FDA approval in 2003 through discontinuation of sales in February 2014 due to low use and suggested that a possible reason for low use could be the treatment protocol. Multiple visits were needed for dosimetry, in order to determine the administered activity to maximize tumor response and minimize toxicity. Also, the $\mathrm{mAb}$ was labeled with ${ }^{131} \mathrm{I}$, which could result in thyroid exposure, necessitating premedication. The protocol for ${ }^{90}$ Y-ibritumomab tiuxetan administration is less involved because dosimetry is not required, the therapy can be given on an outpatient basis, and it is well tolerated with no myelotoxicity at the dose ranges that have been studied. However, Dr. O'Malley noted that, despite data supporting radioimmunotherapy and its inclusion in National Comprehensive Cancer Network Guidelines, few oncologists prescribe ${ }^{90}$ Y-ibritumomab tiuxetan.

Dr. O'Malley concluded her talk with a review of challenges and opportunities for radioimmunotherapy in lymphoma. Large randomized trials are difficult to design, given the rapidly changing available pharmaceuticals, but would bolster existing data. Reimbursement issues need to be addressed, and the misperception that radioimmunotherapy is more expensive than chemotherapy needs to be corrected. Increasing personnel trained to administer radioimmunotherapy could lead to improved access, and advances in dosimetry could maximize the ability to administer treatment early and effectively. Research should be done on additional targets beyond CD20, new labeling agents, and improved antibodies.

\section{Peptide Receptor Radionuclide Therapy (PRRT) of Neuroendocrine Tumors (NETs)}

Dr. Richard Baum (Zentralklinik Bad Berka) discussed PRRT in NETs of the pancreas and midgut.

NETs are the second most common gastrointestinal cancer, and although the incidence is low, prevalence is high: approximately 120,000 cases in the United States, 296,000 in Europe, and 2.4 million worldwide. Current FDA-approved therapies for metastatic NET (G1/G2) include somatostatin analogs and tyrosine kinase inhibitor/mammalian target of rapamycin pathway inhibitors at a cost of approximately $\$ 4,000$ per month. Somatostatin analogs do not produce a complete response and rarely result in partial response. The complete response rate for mammalian target of rapamycin pathway inhibitors is low and may be associated with significant toxicity, including drug-induced death (11-13).

Dr. Baum presented findings from the European Neuroendocrine Tumor Society Center of Excellence at Zentralklinik Bad Berka and from German multiinstitutional registry studies. He noted that PRRT improves PFS and overall survival by several years in metastatic or progressive G1-G2 NETs compared with other treatment modalities regardless of previous therapy. Further, the combination of ${ }^{177} \mathrm{Lu}-$ and ${ }^{90}$ Y-based PRRT (duo PRRT) may be more effective than either radionuclide alone. Thus, in patients with progressive NETs, fractionated, personalized PRRT with lower doses of radioactivity given over a longer period (Bad Berka Protocol) may result in an excellent response even in advanced cases.
A German multiinstitutional registry study with prospective follow-up in 450 patients also indicates that PRRT is an effective therapy for patients with G1-G2 NETs, irrespective of previous therapies, with a survival advantage of several years compared with other therapies and only minor side effects. Median overall survival of all patients from the start of treatment was 59 mo. Median PFS measured from the last cycle of therapy was 41 mo. Median PFS of pancreatic NET was 39 mo. Similar results were obtained for NET of unknown primary (median PFS, $38 \mathrm{mo}$ ) whereas NET of the small bowel had a median PFS of 51 mo. Side effects such as nephro- or hematotoxicity were observed in only $0.2 \%$ and $2 \%$ of patients, respectively.

Patient selection for personalized PRRT (Bad Berka Score) takes into account a multiplicity of factors including standardized uptake values on PET/CT, renal function, hematologic status, liver involvement, extrahepatic tumor burden, and Ki-67 index. More than $9,000{ }^{68} \mathrm{Ga}$ PET/CT clinical studies have been done at Bad Berka since 2004. In June 2014 the European Medicines Agency approved for the first time a ${ }^{68} \mathrm{Ga}$ generator, and 8 different ${ }^{68} \mathrm{Ga}-$ labeled radiopharmaceuticals are now in clinical use at the center. Dr. Baum underscored the advantages of ${ }^{68} \mathrm{Ga}$-somatostatin PET/CT (e.g., using DOTATOC, DOTATATE, or DOTANOC) to determine which patients are most likely to benefit from radionuclide therapy.

Experience with PRRT in Europe dates to 1994, when PRRT with high-dose ${ }^{111}$ In-octreotide was first performed by Krenning's group in Rotterdam (14). The concept has since expanded to centers throughout the world with no commercial support. Established in 2011, the European Neuroendocrine Tumor Society Center of Excellence at Zentralklinik Bad Berka now has more than 1,200 patient visits and administers more than 500 cycles of PRRT every year. Ten physicians at the Center and a dedicated multidisciplinary team of experts in internal medicine; endocrinology; gastroenterology; oncology; abdominal, thoracic, spinal, and heart surgery; radiology; and interventional radiology are directly involved in therapy. An individual treatment plan based on a tumor board consensus is developed for each patient. Four to 6 cycles of PRRT with low to intermediate dosages of radioactivity, and as many as 10 cycles, are administered. For some patients, ${ }^{90} \mathrm{Y}$ and ${ }^{177} \mathrm{Lu}$ are used in combination, given sequentially or concurrently (tandem PRRT). An intraarterial route is used to selectively target liver metastases and large, inoperable primary tumors. All clinical data on PRRT are entered in a prospective clinical database, which now includes more than 1.4 million datasets.

In the Bad Berka experience, which includes treatment of more than 1,251 patients with more than 4,000 therapy cycles, up to 10 cycles of PRRT given over several years were well tolerated by most patients (15). With nephroprotection using amino acids, severe renal toxicity can be avoided or reduced. Hematologic toxicity is usually mild to moderate (except for rare cases of myelodysplastic syndrome, which occur in 2\%-3\%). Although cure of NETs is rarely possible with PRRT, $85 \%$ of patients have improvement in clinical symptoms and $95 \%$ of underweight patients gain $5 \%$ or more of their body mass. In addition, neoadjuvant PRRT can be administered in cases of inoperable NET to render the tumor operable by inducing radiation necrosis, leading to decreased tumor size. In concluding his talk, Dr. Baum emphasized that PRRT should be done at specialized centers because NET patients need individualized interdisciplinary treatment and long-term care. PRRT can be effectively combined with transarterial chemoembolization, radiofrequency ablation, 
chemotherapy (e.g., using capecitabine/5-fluorouracil, temozolomide, or doxorubicin), and kinase inhibitors (e.g., everolimus).

\section{Prostate Cancer Therapy}

Dr. Fatima Karzai (NCI) presented an overview of prostate cancer therapy, highlighting the role of TRT.

Prostate cancer is the most common malignancy in men, with a lifetime risk of 1 in 6 . Age and family history are contributory, and genetic predisposition may play a role in 5\%-10\% of cases. Distant metastases (most commonly to bone) are present in $4 \%$ of cases at diagnosis, and more than $20 \%$ of men with prostate cancer will die of the disease. Clinical parameters such as prostatespecific antigen, Gleason score, and time from surgery to biochemical recurrence are used to stratify patients at high risk of cancerspecific mortality after radical prostatectomy.

The changing landscape of prostate cancer treatment in recent years was reviewed, including the use of abiraterone, enzalutamide, and ${ }^{223} \mathrm{RaCl}_{2}$ (an $\alpha$ emitter with a half-life of $11.4 \mathrm{~d}$, also known as Xofigo [Bayer Healthcare Pharmaceuticals]). Dr. Karzai discussed the need for patient selection to tailor treatment for localized prostate cancer (i.e., watch and wait, androgen deprivation therapy, prostatectomy), locally advanced disease (i.e., prostatectomy or radiotherapy with androgen deprivation therapy), and metastatic disease. The role of androgen deprivation with surgical castration or administration of luteinizing hormone-releasing hormone agonists, antagonists, or antiandrogens; chemotherapy; external-beam radiation therapy (EBRT); and immunotherapy was discussed. The different FDA-approved drugs for metastatic castration-resistant prostate cancer (mCRPC) therapy, including docetaxel, sipuleucel-T, abiraterone, cabazitaxel, enzalutamide, and ${ }^{223} \mathrm{RaCl}_{2}$, were reviewed. Docetaxel is associated with increased median survival, quality of life, and pain response in men with mCRPC, but adverse effects include central nervous system toxicity (20\%-58\%), neutropenia (84\%-99\%), and pulmonary reactions $(41 \%)$. Sipuleucel-T, an active cellular immunotherapy, has a median survival benefit of 4.1 mo with adverse effects that include cerebrovascular events in $3.5 \%$ of patients (16). Abiraterone is a CYP7 inhibitor that blocks androgen synthesis by the adrenal glands, testes, and prostate cancer cells. In clinical trials, an overall survival benefit of 14.8 mo versus 10.9 mo for placebo was demonstrated. Adverse effects include edema (25\%-27\%), fatigue (39\%), and lymphocytopenia (38\%). Cabazitaxel, a novel taxane, has shown improved PFS and overall survival in mCRPC compared with mitoxantrone (2.8 and 15.1 mo vs. 1.4 and $12.7 \mathrm{mo}$, respectively). Adverse effects include fatigue (37\%), anemia (98\%), and neutropenia (94\%). Enzalutamide is a small-molecule androgen receptor antagonist that prevents nuclear translocation and coactivator recruitment. In a phase III study, median survival was 18.4 mo for enzalutamide versus 13.6 mo for placebo. Adverse effects include peripheral edema (15\%), fatigue (51\%), and neutropenia (15\%). Radionuclides have an established role in palliation of metastases from prostate cancer. In phase III trials, ${ }^{153} \mathrm{Sm}$ (a $\beta$ and $\gamma$ emitter) and ${ }^{89} \mathrm{Sr}$ (a $\beta$ emitter) have demonstrated safety and efficacy for pain palliation in patients with mCRPC, and ${ }^{223} \mathrm{RaCl}_{2}$ (received FDA approval in 2013) has been shown to improve overall survival (17). In the ALSYMPCA phase III trial, median survival for men with mCRPC treated with ${ }^{223} \mathrm{RaCl}_{2}$ was $14.9 \mathrm{mo}$, versus $11.3 \mathrm{mo}$ for placebo $(P<0.001)$. Adverse effects associated with ${ }^{223} \mathrm{RaCl}_{2}$ include leukopenia (35\%), thrombocytopenia (31\%), and neutropenia $(18 \%)$.
Dr. Karzai concluded her remarks with recommendations on future directions, noting that this is an exciting time to be treating prostate cancer given the rapidly changing landscape of available medications. For example, results from the CHAARTED trial, presented at the 2014 meeting of the American Society of Clinical Oncology, showed a survival impact for androgen deprivation therapy plus docetaxel versus androgen deprivation therapy alone for hormone-sensitive newly metastatic disease, altering the standard of care for these patients (18). However, an understanding of mechanisms of resistance to drugs such as docetaxel is of importance in improving outcomes for men with metastatic prostate cancer. Studies are needed to elucidate ways to sequence available agents and determine novel strategies for therapy to overcome resistance.

\section{OPPORTUNITIES}

\section{Targets and Targeting Agents}

Dr. Janice Reichert (Reichert Biotechnology Consulting LLC) presented an overview of TRT based on business intelligence research available as of September 2014. Her presentation focused on 4 questions: Who is developing relevant biosimilar antibodies that might be amenable to a radiolabeled form? Which TRTs under development use mAbs effective in a cold form? Which cold mAbs have failed in phase II or III trials because of lack of efficacy? Which mAbs have already been labeled with a radionuclide?

Approximately 200 antibodies are currently under investigation in the United States for cancer, $40 \%$ of which are noncanonical and have undergone extensive protein engineering and design to enhance functionality. Every year, approximately 50 novel antibodies enter clinical studies in the United States. In addition, some patents for antibody therapeutics first approved in the late 1990s and early 2000s have expired, and thus biosimilar versions of these products are also in clinical development. Three biosimilar mAbs relevant for TRT-bevacizumab, rituximab, and trastuzumab-are in phase III studies with expected completion dates ranging from 2015 to 2017.

Half the clinical trials with novel antibodies are in oncology, and many are terminated because of lack of efficacy. Examples of phase II trial failures include carlumab in prostate cancer, dacetuzumab in diffuse large B-cell lymphoma/chronic lymphocytic leukemia, tigatuzumab in breast cancer, and tovetumab in glioblastoma and non-small cell lung carcinoma (NSCLC). The most recent phase III trial failure was with a fusion protein onartuzumab for gastric cancer; other examples include farletuzumab in ovarian cancer, figitumumab in lung cancer, zalutumumab in head and neck cancer, and zanolimumab in T-cell lymphoma.

Cold molecules currently in phase III studies for which radiolabeling could be considered include bavituximab (NSCLC) and elotuzumab (multiple myeloma). Rilotumumab is being studied commercially (Amgen; gastric cancer) as well as in the government and nonprofit sectors (NCI; NSCLC).

Nine radiolabeled $\mathrm{mAbs}$ relevant for TRT are currently in commercially supported studies. Two are in phase I development $\left({ }^{90} \mathrm{Y}-\mathrm{OTSA} 101,{ }^{212} \mathrm{~Pb}\right.$-trastuzumab [trastuzumab]), 6 are in phase I/II or phase II development $\left({ }^{177} \mathrm{Lu}-\mathrm{DOTA}-\mathrm{HH} 1\right.$ [tetulomab], ${ }^{90}$ Y-IMMU-102 [epratuzumab], ${ }^{225} \mathrm{Ac}-$ huM195 [lintuzumab], ${ }^{131}$ I-chTNT-1/B [chTNT], ${ }^{131}$ I-BC8 [BC8], ${ }^{177}$ Lu-ATL-101 [J591]), and one is in phase III development ( ${ }^{90}$ Y-IMMU-107 [clivatuzumab]). Four mAbs - trastuzumab, epratuzumab, lintuzumab, and 
J591-have been studied in cold as well as radiolabeled forms. Adding a radionuclide is only one of many ways to enhance the functionality of mAbs in oncology. Other strategies being tested in clinical studies include glycoengineering and development of antibody-drug conjugates and bispecific antibodies.

Dr. Reichert also urged consideration of molecules in development that are not antibodies, such as the peptibody trebananib, which targets and binds to Ang-1 and Ang-2 and is in a phase III trial for ovarian cancer.

Dr. Reichert concluded her talk with a review of mAbs that incorporate diagnostic radionuclides and a perspective on the best sources of mAbs. The only diagnostic mAb incorporating a radioisotope still on the U.S. market is ${ }^{111} \mathrm{In}$-capromab (ProstaScint;

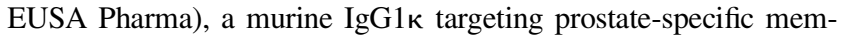
brane antigen. Biosimilar antibodies are the best sources of mAbs for TRT, with trastuzumab, bevacizumab, and rituximab projected to enter the market in the European Union or United States in several years. Many options also exist among the cold mAbs that have failed in phase II or III for efficacy or are currently in phase III, with the choice dependent on the target.

\section{Radiochemistry}

Dr. Martin Brechbiel (NCI) described the range of available radionuclides and the conjugation chemistry needed for TRT applications.

Most radionuclides are metals, and the importance of choosing an appropriate chelating agent was emphasized. A discussion on classic coordination chemistry criteria ensued, and the importance of considering cavity size versus ionic radius, denticity, donor group character, formation kinetics, and dissociation rates was presented. Dr. Brechbiel suggested that the most effective and easiest way to evaluate a chelating agent is with in vivo biodistribution studies. In vitro studies of thermodynamic stability, acidcatalyzed dissociation, and serum stability to predict failure, also can be performed. Although acyclic compounds such as diethylenetriaminepentaacetic acid tend to have faster formation kinetics and form at room temperature, macrocyclic compounds such as DOTA are more stable but require heating.

A detailed discussion of radionuclides and chelating agents followed, starting with actinium and ending with zirconium. For example, it was noted that actinium is being studied in multicenter phase III trials using different DOTAs, although the radiolabeling efficiency is poor $(19,20)$. Several chelating agents exist for ${ }^{212} \mathrm{Bi}$ and ${ }^{213} \mathrm{Bi}$; however, the clinical use of these radionuclides is challenging because of their short half-lives. Coordination chemistry for copper is well established, whereas for thorium it could be improved. Radiolabeling of ${ }^{89} \mathrm{Zr}$ with desferrioxamine has been studied but is not highly stable, as demonstrated by localization of the uncomplexed element in bone (21).

In concluding his remarks, Dr. Brechbiel suggested that most metallic radionuclides of interest for TRT have adequate chelation chemistry but that there are opportunities to fine-tune the science. CHX-A diethylenetriaminepentaacetic acid has proven suitable for in vivo use with ${ }^{111} \mathrm{In},{ }^{86} \mathrm{Y},{ }^{90} \mathrm{Y},{ }^{177} \mathrm{Lu},{ }^{212} \mathrm{Bi}$, and ${ }^{213} \mathrm{Bi}$, whereas C-DOTA and other DOTAs are suitable for ${ }^{111} \mathrm{In},{ }^{86} \mathrm{Y},{ }^{90} \mathrm{Y},{ }^{177} \mathrm{Lu}$, ${ }^{225} \mathrm{Ac}$, and ${ }^{227} \mathrm{Th}$. C-NOTA and other NOTAs are suitable for ${ }^{67} \mathrm{Ga},{ }^{68} \mathrm{Ga},{ }^{111} \mathrm{In},{ }^{64} \mathrm{Cu}$, and ${ }^{67} \mathrm{Cu}$. Desferrioxamine can be used in vivo with ${ }^{89} \mathrm{Zr}$, and sarcophagines are suitable for use with ${ }^{64} \mathrm{Cu}$ and ${ }^{67} \mathrm{Cu}$. Above all, Dr. Brechbiel noted, the choice of chelating agent must be tailored to the TRT application and radionuclide being used.

\section{Radioisotopes and Treatment Planning}

Dr. Barry Wessels (University Hospitals of Cleveland) described new methods of evaluating dose-response relationships for combination therapies, based on lessons learned from EBRT. Radiobiology as a game changer in maximizing the time-averaged therapeutic ratio for successful radioimmunotherapy was emphasized.

The principles of molecular radiobiology were reviewed, and the availability of therapeutic radionuclides and associated emission characteristics were discussed. It was noted that availability has not changed substantially in nearly 30 years. The choice of radionuclide for TRT should take into consideration the emitted particle range, carrier uptake nonuniformity, amount of target radiation delivered, and time-averaged therapeutic ratio as a function of physical and biologic half-life. The utility of small-animal SPECT was reviewed. With $\alpha$-particle dosimetry, the mean dose to macroscopic target volume is not predictive of biologic effect, the mean dose to marrow is not predictive or indicative of therapeutic effect, and the dose to cells is dependent on the spatial distribution of emitters relative to the target cell population.

The importance of dosimetry and development of a suitable treatment plan as with EBRT was emphasized. A computerized tool for multicellular dosimetry (MIRD cell, version 2.0) was highlighted (22). Principles of human dosimetry were discussed, underscoring the tenet that calculated dose is not equivalent to biologic dose. It was noted that radiation quality, subcellular distribution, radiosensitivity, dose rate, repair, and repopulation all contribute to absorbed dose. The biologically effective dose quantifies the biologic effect of a radiation therapy. For radioimmunotherapy, fractionation schemes can be converted to biologically effective dose values in grays, which can then be added or subtracted and converted back to biologically equivalent dose schemes.

Turning to advances in dosimetry, Dr. Wessels described SPECT guidance, studies of spheres for selective internal radiation therapy, and patient-specific 3-dimensional radiobiologic dosimetry (3D-RD). It was suggested that SPECT may be helpful for TRT planning. Also, ${ }^{90} \mathrm{Y}$ PET has been used for quantification of liver dosimetry in selective internal radiation therapy. In a pediatric patient with thyroid cancer and lung metastases, more aggressive therapy with ${ }^{131} \mathrm{I}$ is possible using treatment planning with ${ }^{124} \mathrm{I}$ PET-based 3D-RD. A retrospective analysis of conventional dosimetric methodologies yielded absorbed dose estimates consistent with 3D-RD, but the necessary corrections might not have been known without use of the technology in this case.

The advantage of EBRT combined with TRT versus a single agent was discussed. Use of 2 different modalities together could increase the total tolerated radiation dose because each modality is associated with different nontarget organs at risk. Clinical applications could include, among others, paraganglioma $\left({ }^{131}\right.$ I-metaiodobenzylguanidine) and lesions of the bone ( ${ }^{153} \mathrm{Sm}$-ethylenediaminetetramethylene phosphonate), brain ( ${ }^{131} \mathrm{I}$-radretumab), and liver ( ${ }^{90}$ Y-microspheres) (23).

In concluding his remarks, Dr. Wessels noted that newer methods of combining more accurate dosimetric information with radiobiologic models have led to an increased understanding of TRT dose-response data. Radioimmunotherapy patients are often undertreated because of fear of side effects. Application of the biologically effective dose and equivalent uniform dose radiobiologic model to TRT has been reasonably successful. Using the model within clinically relevant limits can provide guidance on dose-response effects for new fractionation schemes, combination therapy, and clinical trial design. 


\section{Nuclear Medicine}

Dr. Steven Larson (Memorial Sloan Kettering Cancer Center) reviewed the current status and promise of TRT from the perspective of a nuclear medicine physician. Research at the Memorial Sloan Kettering Cancer Center on a theranostic approach to solid tumors, in particular thyroid cancer, was described.

${ }^{131} \mathrm{I}$ is often used for ablation of well-differentiated thyroid cancer; however, in many patients, the disease progresses after ablation, possibly because of low uptake. There is a high prevalence of $B R A F$ mutations in thyroid cancer refractory to treatment with ${ }^{131} \mathrm{I}$, and inhibition of the oncogene may increase uptake. In a study by Memorial Sloan Kettering Cancer Center researchers, a novel theranostic approach was used in a group of thyroid cancer patients who were refractory to ${ }^{131} \mathrm{I}$ therapy, whereby ${ }^{124} \mathrm{I}$ was used to select those patients $(\sim 50 \%)$ whose radioiodine uptake could be restored by treatment with selumetinib (a mitogen-activated protein kinase and mitogen-activated protein kinase kinase 2 inhibitor) over a 4-wk treatment. Patients with dramatic improvement in radioiodine uptake (as predicted by ${ }^{124}$ I PET) were treated, and 5 of 7 had RECIST-based responses to ${ }^{131}$ I therapy of their thyroid malignancy (24). This is an example of precision medicine in which patients who responded could be treated with radioiodine with therapeutic benefit whereas patients with a minimal response could be directed to other therapies and avoid ineffective ${ }^{131}$ I treatment and side effects. Tumors with $R A S$ mutations were particularly susceptible to this strategy.

Moving beyond applications in thyroid cancer, Dr. Larson reviewed work by Memorial Sloan Kettering Cancer Center researchers on theranostic agents for imaging and treating other solid tumors. Radiolabeled 3F8 and 8H9 have been studied in recurrent neuroblastoma. Compartmental intrathecal antibody-based radioimmunotherapy has shown promise in metastatic central nervous system neuroblastoma. Preclinical research is ongoing on multistep targeting, whereby bispecific antibodies serve as targeting vectors for pretargeted radioimmunotherapy (25).

Dr. Larson concluded by emphasizing the potential of theranostics to improve patient selection for radioimmunotherapy and predict those likely to enjoy a meaningful response with minimal toxicity. For thyroid cancer, the goal, given the methods currently available, should be cure, not just disease control.

\section{HURDLES AND INCENTIVES}

\section{Regulatory Affairs}

Dr. Paula Jacobs (NCI) reviewed regulatory requirements for submitting an Investigational New Drug (IND) application to the U.S. FDA (26), noting that other countries have similar but not identical requirements and regulatory processes. The elements of an IND were explained, and special issues for metal-containing drugs were described.

The purpose of an IND is to gain exemption from the law requiring an approved marketing application for a drug before transport or distribution across state lines. An IND should be prepared after preliminary in vivo pharmacology and toxicity studies have been completed in animal models. The FDA's assessment of an IND is designed to ensure that a clinical protocol is not unnecessarily risky for the patient population in which it is being tested. The agency has 30 days to review the submission, and applicants can expect to receive "requests for information" that require an immediate response.

Three categories of information are required in an IND: data from animal pharmacology and toxicology studies; specifics on chemistry, manufacturing, and controls; and a detailed protocol for the clinical study. The preclinical data are reviewed for safety, and the clinical investigator's qualifications are evaluated. Efficacy is a secondary concern in early trials. It is important to remember that INDs are "living" documents, that is, an annual report is required, adverse events must be reported, and protocol amendments as well as new protocols must be submitted to the FDA. Investigators submitting INDs are expected to understand the FDA guidance regarding documents and provide a coherent and defensible biologic rationale. Preclinical data must document bioactivity in vitro and in vivo; absorption; distribution, metabolism, and excretion; and toxicology. For radiopharmaceuticals, dosimetry is required. The clinical plan must be relevant and supportable, with a strong emphasis on patient safety, and provide the framework for future research.

The regulatory path is the same for small molecules, most biologicals, and radiolabeled drugs, but the strategy used to apply for an IND may differ depending on the compounds involved, pharmacokinetics, pharmacodynamics, and dose escalation. A new protocol can be filed under an existing IND. Radioactive drugs that have been used in humans but will be studied in a research protocol that does not involve therapy can be approved by an FDA radioactive drug research committee. If a new IND is filed with a letter of agreement associated with another IND, only new information (not in the previously approved IND) need be submitted. All sections must be completed for new, standalone INDs.

Most traditional INDs are investigator-initiated and use a single agent that is expected to proceed to phase III trials and a new drug application (NDA). Extensive preclinical data typically are required, and dose escalation is involved. With an exploratory IND (x-IND, phase 0 ), up to 5 agents can be explored under a single IND for microdose studies designed to evaluate pharmacokinetics or specific target imaging but not therapeutic effects. A microdose is defined as less than 1/100th of the dose calculated to yield a pharmacologic effect and less than $100 \mu \mathrm{g}$ of a small molecule or less than $30 \mathrm{nmol}$ of a protein. Fewer preclinical data are expected, and resubmission as a traditional IND is required to continue development if studies done under an X-IND are successful. Of note, an x-IND can also be called an e-IND, which unfortunately is also the terminology for an emergency IND.

Dr. Jacobs provided links to FDA guidance on the IND process and noted that all the information required for an IND except the clinical aspects can be "imported" from another IND by a letter of reference. Details were provided about components of the chemistry, manufacturing, and controls; pharmacology and toxicology; and in vitro and in vivo testing sections. Information required for metal-containing drugs includes data on exchange with biologic metals and protein binding and absorption, distribution, metabolism, and excretion of all components. Robust analytic techniques are necessary to quantify both metal and organic components in biologic matrices. Special concern exists about retained components and metabolites.

It was noted that toxicology studies can be a major barrier to IND filing. FDA guidance typically demands 2 species and multiple studies, which can be expensive, lengthy, and require a lot of material. However, the initial program is negotiable early in the IND process if the proposed program is supported by good science that ensures patient safety. Special characteristics that inform nonsafety evaluations are mass dose; route of administration; frequency of use; proposed test population; biologic, physical, and effective half-lives; data on similar drugs; and specific FDA guidance for the specific drug class. 
Dr. Jacobs concluded her remarks with a regulatory affairs perspective on ${ }^{223} \mathrm{Ra}$-dichloride. In vitro studies in the ${ }^{223} \mathrm{Ra}$ dichloride NDA documented survival, DNA damage, cell cycle effects, and osteoclast effects. In vivo efficacy data were on dose, mechanism of action, and survival in a model. Absorption, distribution, metabolism, and excretion studies were performed in mice, rats, and dogs. Toxicology studies were performed in mice, rats, and dogs plus local irritation in rabbits. No genotoxicity or reproductive toxicity studies were required.

Dr. Jacob's final message to investigators preparing INDs for the first time was to seek help from someone with experience in the process.

\section{Industry}

Dr. David Goldenberg (Immunomedics) provided a personal perspective on hurdles and opportunities for TRT in oncology. Lessons learned from ${ }^{131}$ I-tositumomab and ${ }^{90}$ Y-ibritumomab tiuxetan and experience with studies of ${ }^{90}$ Y-radiolabeled clivatuzumab tetraxetan $\left({ }^{90}\right.$ Y-labeled hPAM4 [clivatuzumab]), which has fast track status with the FDA for treatment of pancreatic cancer, were presented.

The pipeline of radiopharmaceutical candidates has declined, from 25 in 2006 to 4 in 2014, only one of which ( ${ }^{223}$ Ra-dichloride) is approved for therapy. Prospects for growth lie in personalized medicine and identification of companion diagnostics for TRT. Hurdles faced by the field include specificity of biomarkers, complexity and cost of evaluating combined modalities, and proving to regulatory authorities an improvement in disease management. Collaboration between nuclear medicine physicians and oncologists must be improved; the tumor board concept has potential in that regard. For commercial entities, return on investment and time to market are considerations.

The key lesson learned from ${ }^{131}$ I-tositumomab and ${ }^{90}$ Y-ibritumomab tiuxetan is that science and efficacy alone do not guarantee success. An extensive pipeline of therapies is available to oncologists, who are rapidly adopting new modalities such as targeted agents, oral drugs, and combination therapies. To succeed, radiopharmaceuticals must change disease management by distinguishing patients whose tumors are operable versus inoperable and those who will benefit versus will not benefit from specific treatments.

The presentation then turned to the experience with ${ }^{90}$ Y-clivatuzumab, which has been developed as a fractionated therapy for metastatic pancreatic cancer. A phase Ib trial completed in 2014 showed improved efficacy for a combination with fixed, low-dose gemcitabine versus ${ }^{90}$ Y-clivatuzumab alone in third-line therapy. An international phase III, multicenter, double-blind trial is ongoing of ${ }^{90}$ Y-clivatuzumab plus gemcitabine versus gemcitabine alone in that same setting (ClinicalTrials.gov identifier NCT01956812). Patients in the experimental arm are receiving ${ }^{90}$ Y-clivatuzumab 3 times weekly plus gemcitabine 4 times weekly for multiple cycles. Patients in the active comparator arm are receiving placebo 3 times weekly plus gemcitabine 4 times weekly for multiple cycles. In the clinical studies performed to date, no infusion reactions to ${ }^{90} \mathrm{Y}$ clivatuzumab have been seen, myelosuppression was manageable, and no other significant adverse effects were reported. A survival analysis has shown improved overall survival (median, $9.3 \mathrm{mo}$ ) in 31 patients with previously treated metastatic pancreatic cancer who received multiple cycles of ${ }^{90}$ Y-clivatuzumab combined with low-dose gemcitabine. The results suggest that radioimmunotherapy has promise as third-line therapy for pancreatic cancer and that multiple cycles are essential.
Other applications of radiopharmaceuticals being explored by Immunomedics include epratuzumab in B-cell tumors, ${ }^{18} \mathrm{~F}$-AlF $\mathrm{mAbs}$ and peptides as companion imaging agents, pretargeted immuno-PET and radioimmunotherapy as a bispecific antibody platform, and combinations of antibody-drug conjugates with radioimmunotherapy.

\section{Radiopharmaceuticals}

Ms. Sally Schwarz (Washington University) provided an overview of the toxicity testing requirements for INDs submitted to the FDA, which have evolved to mandate additional monitoring, including latent toxicity. The experience with approval of radiotherapeutics from ${ }^{89} \mathrm{Sr}$-chloride in 1993 to ${ }^{223} \mathrm{Ra}$-dichloride in 2013 was discussed. The focus was on FDA guidance on pharmacology and toxicology in 2 species.

Acute toxicity testing, based on administration of one or more doses over a period not to exceed $24 \mathrm{~h}$, has been required for pharmaceuticals since 1996. For intravenous drugs, a single route is sufficient; for all other routes, the intended route plus intravenous must be tested. Two mammalian species must be included, with testing in small groups, observation for $14 \mathrm{~d}$ after administration, and gross necropsies on all animals.

${ }^{89} \mathrm{Sr}$-chloride (Metastron; GE Healthcare) was, in 1993, the first radiopharmaceutical submitted for FDA approval as an adjunct to local-field EBRT in mCRPC. Data from the Trans Canada study showed a statistically significant difference $(P<0.002)$ in the number of new painful sites per patient in the placebo group versus the active group (27). A statistically significant difference also was demonstrated in median time to further radiotherapy in the 2 groups $(P=0.006) .{ }^{89} \mathrm{Sr}$-chloride was shown to be effective at reducing disease progression and need for analgesic support and improving quality of life, with increased but tolerable hematologic toxicity.

Three years later, ${ }^{211}$ At toxicity in $\mathrm{B} 63 \mathrm{CF}$ mice was documented in preparation for designing future investigations of ${ }^{211}$ At-labeled therapeutic compounds. In a study by McLendon et al., up to $7,400 \mathrm{kBq}(200 \mu \mathrm{Ci})$ for $80 \mathrm{~d}$ was administered to one group, up to $2,960 \mathrm{kBq}(80 \mu \mathrm{Ci})$ was used in the second group, and up to $740 \mathrm{kBq}(20 \mu \mathrm{Ci})$ was administered to the third group (28).

More recently, approval in 2013 of ${ }^{223} \mathrm{Ra}$-dichloride, a first-inclass radiopharmaceutical that selectively targets bone metastases, was based on the results of the randomized phase III ALSYMPCA trial. ${ }^{223} \mathrm{Ra}$-dichloride was shown to prolong overall survival (3.6-mo advantage; $P=0.00185$ ) and time to first symptomatic skeletal event $(P=0.00046)$ versus placebo in patients with mCRPC and symptomatic bone metastases, with toxicity comparable to placebo. A phase I clinical trial in breast and prostate cancer with skeletal metastases demonstrated safety and tolerability at all therapeutically relevant dosages. Data from a phase II trial in advanced breast cancer and progressive bone-dominant disease demonstrated targeting of areas of increased bone metabolism and biologic activity.

Turning to trials of radiopharmaceuticals currently under way, the international multicenter Advanced Accelerator Applications (AAA) ${ }^{177}$ Lu-DOTATATE trial was approved on the basis of toxicology studies in rats and dogs. A 23-cGy dose limit to the kidneys was established from experience with AAA ${ }^{177} \mathrm{Lu}-\mathrm{DOTA}-$ TATE in EBRT. A phase III randomized trial of ${ }^{223} \mathrm{RaCl}_{2}$ plus abiraterone acetate in mCRPC was begun in March 2014. Subjects in the active arm receive ${ }^{223} \mathrm{RaCl}_{2}(50 \mathrm{kBq} / \mathrm{kg}$ intravenously) every 
$4 \mathrm{wk}$ for 6 cycles plus 1,000 mg of oral abiraterone acetate daily plus prednisone/prednisolone until an on-study symptomatic skeletal event occurs. The estimated study completion date is July 2020.

Commenting on the cost of toxicology studies, Ms. Schwarz noted that a single rodent study can cost $\$ 70,000-\$ 120,000$ and that large-animal models such as swine may be a cheaper alternative that would allow performance of toxicology studies in a single species for a therapeutic IND. A good-laboratory-practice lab should be used. Late toxicity can be determined using EBRT, with latent periods of 3-7 mo for nephritis in rats and 10 mo for renal dysfunction in dogs. Rats and dogs are species appropriate for assessing pulmonary fibrosis, and rabbits and dogs for myocardial fibrosis. Human dosimetry and pharmacokinetics using tracer doses should be determined before initiation of a late radiation toxicity study. Timing of toxicity studies can vary on the basis of risk and benefit, but completion before phase II dose escalation is recommended. The highest mass dose of the nonradioactive compound should be used as the control, and at least 4 dose levels should be included to identify dose-related toxicity.

In concluding her presentation, Ms. Schwarz noted that for TRT to move forward more rapidly, FDA hurdles must be identified and researchers must work together to share toxicity data and possibly use the $\mathrm{x}$-IND concept for radiotherapeutics.

\section{ROUNDTABLE DISCUSSION}

Dr. Ulli Köster (Institut Laue-Langevin) and Dr. John Valliant (Centre for Probe Development and Commercialization, McMaster University) led a roundtable discussion with experts Aimal Khan Ahmed (Bayer Healthcare), Jehanne Gillo (Department of Energy), Andreas Kluge (ABX-CRO), Deepa Narayanan (NCI Small Business Innovation Research [SBIR]), and Greg Evans (SBIR). The participants were invited to offer their opinions on a series of questions posed by the cochairs.

The experts were asked, given the state of the field today, what information would need to be presented to a pharmaceutical company to secure an investment in TRT. The panel's consensus was that there is no easy answer, considering the experience with ${ }^{131}$ I-tositumomab and ${ }^{90}$ Y-ibritumomab tiuxetan and the need for a return on investment. On a positive note, the opinion was shared that ${ }^{223} \mathrm{RaCl}_{2}$ is likely to play a major role in the oncology portfolio at Bayer Healthcare, given the company's acquisition of Algeta. Licensing of hospitals to administer the product was considered a possible postapproval barrier to adoption of ${ }^{223} \mathrm{RaCl}_{2}$. In this ongoing process, already over 1,400 hospitals have been licensed. This effort can pave the way for future clinical application of other TRT with $\alpha$ emitters. Inadequate reimbursement, given the complexity of the treatment, also is a concern for TRT in general.

TRT as a component of NCI SBIR's funded small-business portfolio then was discussed. The portfolio includes approximately 10-20 active projects involving radionuclides for imaging, versus fewer than 5 for therapy, but there is interest in expanding the offerings. Congress has recently reauthorized the SBIR and Small Business Technology Transfer programs and increased the set-aside percentage for funding of them, in the context of the entire NCI budget.

Only U.S. small business concerns can participate in the SBIR program (http://sbir.cancer.gov/about/eligibility/). The NCI's support for a robust TRT pipeline and encouragement of out-of-thebox thinking to advance the field were emphasized.

The Department of Energy's National Isotope Development Center (http://www.isotopes.gov/) and its role in facilitating production of isotopes and ensuring their availability for medical research were reviewed. The National Isotope Development Center works closely with a representative from the National Institutes of Health to determine the needs of the Institutes for isotopes.

\section{CONSENSUS}

Breakout sessions held as part of the NCI-SNMMI workshop included cross-sections of stakeholders and focused on 1 of 4 disease-specific topics: breast cancer, lymphoma and leukemia, lung cancer and neuroendocrine cancer, or prostate cancer. Summaries of the discussions and consensus reached by each group were presented by a rapporteur with a focus on the current state of the art of TRT, clinical indications, apparent strengths and weaknesses, the most promising advances in the field, and challenges to acceptance and incorporation into routine clinical use.

\section{Breast Cancer}

Dr. Stanley Lipkowitz (NCI) summarized the results of the breakout group focusing on breast cancer.

The logistics of treatment execution were discussed, and several hurdles to the adoption of TRT were reviewed. For example: short half-lives make radionuclide transportation difficult, protocols requiring dosimetry result in multiple patient visits, coordination of multidisciplinary teams is complicated, loss of revenue and risks associated with radiation are concerns, and competition from other agents works against use of radionuclides in clinical trials and in the commercial setting.

Past experience with TRT suggests that success depends on an effective multidisciplinary team approach. Opportunities exist for radioimmunotherapy in breast cancer. Although there are targeted agents for estrogen receptor-negative and HER2-positive breast cancer, TRT could have a niche as a second- or third-line therapy. Also, the success of current breast cancer therapy has led to increased survival; however, the incidence of brain metastases has also increased. Intrathecal TRT may have an advantage over chemotherapy in this setting.

In conclusion, the breakout group felt the best path forward for TRT in breast cancer was to address unmet therapeutic needs and to encourage the development of multidisciplinary teams to support its administration and management. Research is needed to identify whether the best targeting agents for breast cancer are radiolabeled antibodies, fragments, or peptides.

\section{Lymphoma and Leukemia}

Dr. Jeffrey Norenberg (University of New Mexico) presented the results from the breakout group focusing on lymphoma and leukemia.

There are several opportunities for TRT in lymphoma and leukemia. For example, TRT could be helpful in T-cell lymphoma or in bone marrow conditioning or myeloablation before autologous bone marrow transplantation. In leukemia, surface molecules are well characterized and could present potential targets for radioimmunotherapy. Combinations of EBRT and TRT have yet to be studied in clinical trials.

The breakout group discussed the need for development of an economic model to sustain TRT, including well-described roles for oncologists, nuclear medicine physicians, and radiation oncologists; increased interdisciplinary communication; and transparency regarding cost and availability. The need to bolster patient education about available treatment options was mentioned. 
Structured training should be made available to all providers involved in radionuclide therapy, including medical oncologists, radiation oncologists, radiochemists, and trainees, and focused specialty meetings should be convened, including cooperation with several professional groups such as the American Society for Radiation Oncology, SNMMI, and American Society of Clinical Oncology. A change in the paradigm at the FDA for INDs and NDAs regarding toxicity of radionuclides versus nonradioactive therapies is needed to fully realize the potential of TRT, and increased engagement of patient advocacy groups could help influence the FDA and payers in that regard.

\section{Lung Cancer and Neuroendocrine Neoplasms}

Dr. Shakun Malik (NCI) reported on the breakout group discussion regarding lung and neuroendocrine cancers.

Opportunities exist for epidermal growth factor receptor TRT in lung cancer. Two drugs that target the epidermal growth factor receptor subfamily (erlotinib and afatinib) are approved for firstline therapy of advanced NSCLC. Crizotinib and ceritinib are being studied to target the anaplastic lymphoma kinase gene in NSCLC. Covalent (irreversible) epidermal growth factor receptor inhibitors are being studied to target mutant forms while sparing wild-type, or normal, epidermal growth factor receptor. Most are small fluorinated or chlorinated molecules, suggesting the potential for radiolabeling with isotopes such as ${ }^{18} \mathrm{~F}$ or ${ }^{11} \mathrm{C}$. This strategy would facilitate companion diagnostic imaging and therapy and might enhance potency. Other targets in NSCLC are TTF1 (a specific marker for primary lung adenocarcinoma) and carcinoembryonic antigen. A preliminary study suggests that radiolabeled MOC-31 may be useful in diagnosis and therapeutic management of small cell lung carcinoma (29). The results of a phase I doseescalation study of ${ }^{188}$ Re-P2045 for TRT in patients with NSCLC or small cell lung carcinoma suggest that further research is warranted (30).

Opportunities exist for TRT in NETs. PRRT with ${ }^{177} \mathrm{Lu}$ - and ${ }^{90}$ Y-octreotide derivatives has been validated in Europe in NETs, as described in Dr. Baum's presentation. Improvements in dosimetry may facilitate FDA approval of clinical trials for new $\alpha$ and $\beta$ emitters.

The breakout group concluded that the future for TRT in lung cancer and NETs is likely to be in dosimetry as a way to personalize therapy, a theranostic approach, research on $\alpha$-particle therapy, and potential for orphan drug status. Challenges to TRT include the need for intravenous access versus the oral route of most non-TRT therapies, issues with availability of good laboratory practice-grade peptides, and the cost of an NDA.

\section{Prostate Cancer}

Dr. Eric Rohren (M.D. Anderson Cancer Center) summarized the discussion of the breakout group focusing on prostate cancer.

Every year, approximately 220,000 men are diagnosed with the disease in the United States. Twenty percent of deaths due to the disease occur in the $5 \%$ of patients who have metastases at diagnosis (http://seer.cancer.gov/statfacts/html/prost.html). Although drug treatments for mCRPC are rapidly expanding, they are having only a limited impact on improving survival curves. Thus, the group saw a significant opportunity for TRT throughout the disease spectrum and particularly in mCRPC.

Hurdles identified for TRT in prostate cancer included tumor heterogeneity, the choice between small molecules and antibodies, the impact of TRT versus other therapies, and the need to increase the profile of TRT among oncologists. Two niches identified for
TRT included treatment of men with metastases at diagnosis and imaging and treatment of primary disease, regional nodes, and metastases. Current techniques for imaging nodal metastases are lacking, and radiopharmaceuticals could help determine which patients should receive aggressive treatment. TRT also may have a role in high-grade tumors, for which therapeutic options are limited, as well as in the adjuvant setting in combination with standard treatments. Experience to date with prostate-specific membrane antigen suggests that it may be the best target for radionuclides such as ${ }^{99 \mathrm{~m}} \mathrm{Tc},{ }^{68} \mathrm{Ga},{ }^{131} \mathrm{I},{ }^{177} \mathrm{Lu}$, and ${ }^{225} \mathrm{Ac}$.

In order for the field to move forward, Dr. Rohren underscored the need to take a theranostic approach to radiopharmaceuticals, that is, to develop individual agents for both diagnosis and therapy, or in combination with imaging agents. An understanding of their mechanism of action, optimization of dosimetry, and demonstrated efficacy are important to gaining increasing support for TRT from oncologists and their patients.

Other barriers to the success of TRT discussed by the breakout group include the limited support for development of therapies from bench through approval to bedside and lack of messaging. Outreach should be done to increase support from all stakeholders-clinicians, patients, advocacy groups, government agencies, and payers - with a focus on data demonstrating efficacy.

\section{HIGHLIGHTS AND FUTURE DIRECTIONS}

Dr. Hossein Jadvar provided an overview of the workshop, emphasizing that the overarching goal of TRT is to deliver timely, cost-effective therapy offering the best possible outcome to patients.

The second NCI-SNMMI workshop expanded on what was accomplished in 2013. The content was disease-focused, and the group of stakeholders was broader, including industry, regulatory, and government colleagues from the United States, as well as European experts.

In the area of preclinical research, optimal combinations of targets, chelates, and radionuclides were discussed. In addition to the existing in vitro and in vivo models, potential exists for validation of new in vivo models in larger animals. Robust, reproducible data are needed, as are dosimetry models for treatment planning in order to comply with regulatory requirements and provide optimal patient care.

Often clinical trials including TRT have suboptimal methodology with nonstandardized endpoint definitions. To advance, appropriately designed large multicenter, randomized trials must be performed. Time is of the essence, and comparison of TRT to current therapies, which are rapidly evolving, is needed. Outcomes such as quality of life should be considered since cancer is increasingly viewed as a chronic disease. TRT may also be helpful early in the course of disease, as in the case of, for example, micrometastases and oligometastatic disease. Novel regimens could include TRT, EBRT, or chemotherapy; multistep targeted chemoradionuclide immunoconjugates; and therapy with different radionuclides in patients who fail initial TRT. The potential for theranostic applications to guide patient selection, identify optimal dose, and assess treatment effectiveness was advocated.

The industry perspective on TRT production and marketing, access to raw materials, regulatory issues, and cost of clinical trials remain concerns. Facilitating dosimetry and TRT implementation is important. Education for patients and providers is needed. The silo mentality of specialties and the inability of specialists to share revenue were identified as impediments to TRT adoption. 
There are several barriers in the area of regulation and reimbursement. In the United States, some radionuclides are difficult to access, some are unapproved, and even those that are approved and reimbursable may be underutilized. Although published reports exist on the use of radionuclides for TRT in Europe, the data rarely can be incorporated into applications for approval and reimbursement in the United States. A need was identified to streamline coordination between the FDA and the Centers for Medicare and Medicaid Services such that reimbursement can keep pace with new drug approvals. Also discussed were issues with levels of reimbursement and a potential need to mandate disclosure by providers to patients of all therapeutic options available to them.

Partnership of all stakeholders was underscored as key to the future of TRT. In terms of the overarching government strategy, coordination is needed among the National Institutes of Health, FDA, Nuclear Regulatory Commission, Centers for Medicare and Medicaid Services, and Congress. In academia, a collaborative approach is needed to raise awareness and knowledge of TRT among all professionals. Pharmaceutical companies should be encouraged to view TRT as an important tool in the armamentarium of cancer therapy. Unbiased and accurate patient information about TRT is needed, and a paradigm shift in the practice of TRT to care by multispecialty teams is important. Inclusion of nuclear medicine specialists on tumor boards was discussed as an initial step toward fostering collaborative practice, as was the importance of outreach.

In conclusion, Dr. Jadvar suggested the need for a balanced, unbiased approach to the integration of TRT into the therapeutic algorithm for patients with cancer. Research must be actively pursued so that barriers to the adoption of TRT can be overcome. The key is to demonstrate that-whether given alone, in combination, or sequentially_-TRT has an important role in patient care.

\section{CONCLUSION}

In summing up, cochair Dr. Zukotynski suggested that the time for TRT is now and that there is a need to bring together professionals from various walks of life to share their expertise on TRT. Dr. Fahey echoed this sentiment and expressed thanks to the workshop organizers and participants for demonstrating their dedication to the specialty. SNMMI's acknowledgment of the importance of TRT is evidenced by its plans to develop a center of excellence that will provide professional networking and educational opportunities in the area while simultaneously serving as a resource for development and implementation of Society policy. The organization also has a role to play in fostering partnerships with organizations such as the American Association of Physicists in Medicine, American Society for Radiation Oncology, and American Society of Clinical Oncology and in collaborating with agencies such as the NCI, FDA, and Department of Energy.

On the basis of the presented discussion and conclusions, the following set of actions is recommended. New research is necessary to determine the most promising approaches for the next generation of targeted radionuclide therapies. This research may involve availability of new raw materials, new or increased supply of therapeutic radionuclides, selection of proper targeting approaches, development of appropriate in vivo models in larger animals, construction of dosimetric models, and, eventually, appropriately designed large multicenter, randomized trials. The ability to fund such research presents a considerable challenge, as do the regulatory issues associated with bringing such novel therapies to the clinic. For these reasons, greater cooperation among all stakeholders is necessary to realize the promises of TRT. Those of different specialties need to work more closely in an open partnership with our international colleagues and industry as well as governmental funders and regulators.

With the 75th anniversary of the first therapeutic use of a radionuclide approaching in 2016, the time may be opportune to increase the profile of TRT.

\section{DISCLOSURE}

No potential conflict of interest relevant to this article was reported.

\section{ACKNOWLEDGMENTS}

The organizing committee is made up of the following individuals: Frederic Fahey (Boston Children's Hospital, Harvard Medical School); Katherine Zukotynski (University of Toronto and Harvard Medical School); Hossein Jadvar (University of Southern California); Jacek Capala (NCI); C. Craig Hill (NCI); Ulli Köster (Institut Laue-Langevin); Jason Lewis (Memorial Sloan Kettering Cancer Center); Frank I. Lin (NCI); and Bhadrasain Vikram (NCI).

The contributors include Richard Wahl (Johns Hopkins University); Ruby F. Meredith (University of Alabama at Birmingham); Cathy S. Cutler (University of Missouri); Lee Allen (Spectrum Pharmaceuticals); Rick Satitpunwaycha (Spectrum Pharmaceuticals); Janis O'Malley (University of Alabama at Birmingham); Richard Baum (Zentralklinik Bad Berka); Fatima Karzai (NCI); Ekaterina Dadachova (Yeshiva University); Janice Reichert (Reichert Biotechnology Consulting LLC); Martin Brechbiel (NCI); Barry Wessels (University Hospitals of Cleveland); Steven Larson (Memorial Sloan Kettering Cancer Center); Aimal Khan Ahmed (Bayer Healthcare); Paula Jacobs (NCI); David Goldenberg (Immunomedics); Sally Schwarz (Washington University in St. Louis); John Valliant (Centre for Probe Development and Commercialization); Jehanne Gillo (Department of Energy); Andreas Kluge (ABX-CRO); Deepa Narayanan (SBIR); Greg Evans (SBIR); Giuseppe Giaccone (Georgetown University); Martin Pomper (Johns Hopkins University); Stanley Lipkowitz (NCI); Jeffrey Norenberg (University of New Mexico); Shakun Malik (NCI); and Eric M. Rohren (M.D. Anderson Cancer Center).

We gratefully acknowledge the contributions of the following individuals to this work: Anne Hiller, Nikki Wenzel-Lamb, Judy Brazel, and Virginia Pappas (SNMMI), as well as Erica Butler, Ling Lang, and Pat Angelis (NCI).

\section{REFERENCES}

1. Fahey F, Zukotynski K, Capala J, et al. Targeted radionuclide therapy: proceedings of a joint workshop hosted by the National Cancer Institute and the Society of Nuclear Medicine and Molecular Imaging. J Nucl Med. 2014;55:337-348.

2. Bryan JN, Bommarito D, Kim DY, et al. Comparison of systemic toxicities of ${ }^{177} \mathrm{Lu}$-DOTMP and ${ }^{153} \mathrm{Sm}$-EDTMP administered intravenously at equivalent skeletal doses to normal dogs. J Nucl Med Technol. 2009;37:45-52.

3. Morschhauser F, Radford J, Van Hoof A, et al. ${ }^{90}$ Yttrium-ibritumomab tiuxetan consolidation of first remission in advanced-stage follicular non-Hodgkin lymphoma: updated results after a median follow-up of 7.3 years from the international, randomized, phase III first-line indolent trial. J Clin Oncol. 2013;31:1977-1983.

4. Shankland KR, Armitage JO, Hancock BW. Non-Hodgkin lymphoma. Lancet. 2012;380:848-857. 
5. Solal-Céligny $\mathrm{P}$, Roy $\mathrm{P}$, Colombat $\mathrm{P}$, et al. Follicular lymphoma international prognostic index. Blood. 2004;104:1256-1265.

6. Seldin DW. Techniques for using Bexxar for the treatment of non-Hodgkin's lymphoma. J Nucl Med Technol. 2002;30:109-114.

7. Ganti AK, Bociek RG, Bierman PJ, et al. Follicular lymphoma: expanding therapeutic options. Oncology. 2005;19:213-228.

8. Chang S, Mukhi N, Wang K, et al. Novel CD20 monoclonal antibodies for lymphoma therapy. J Hematol Oncol. 2012;5:64.

9. Radford J, Van Hoof A, Vitolo U, et al. ${ }^{90}$ Y-ibritumomab tiuxetan (Zevalin) consolidation of first remission in advanced-stage follicular non-Hodgkin's lymphoma: update results after a median follow-up of 66.2 months from the international, randomized, phase III First-Line Indolent Trial (FIT) in 414 patients. Abstract presented at: 53rd ASH Annual Meeting and Exposition; December 1013, 2011; San Diego, California.

10. Schaefer NG, Ma J, Huang P, et al. Radioimmunotherapy in non-Hodgkin lymphoma: opinions of U.S. medical oncologists and hematologists. J Nucl Med. 2010;51:987-994.

11. Raymond E, Dahan L, Raoul JL, et al. Sunitinib malate for the treatment of pancreatic neuroendocrine tumors. N Engl J Med. 2011;364:501-513.

12. Yao JC, Shah MH, Ito T, et al. Everolimus for advanced pancreatic neuroendocrine tumors. N Engl J Med. 2011;364:514-523.

13. Baum RP, Kulkarni HR. Theranostics: from molecular imaging using Ga- 68 labeled tracers and PET/CT to personalized radionuclide therapy-the Bad Berka experience. Theranostics. 2012;2:437-447.

14. Kwekkeboom DJ, Kho GS, Lamberts SW, Reubi JC, Laissue JA, Krenning EP. The value of octreotide scintigraphy in patients with lung cancer. Eur J Nucl Med. 1994;21:1106-1113.

15. Baum RP, Kulkarni HR, Carreras C. Peptides and receptors in image-guided therapy: theranostics for neuroendocrine neoplasms. Semin Nucl Med. 2012;42:190-207.

16. Kantoff PW, Higano CS, Shore ND, et al. Sipuleucel-T immunotherapy for castration-resistant prostate cancer. N Engl J Med. 2010;363:411-422.

17. Parker C, Sartor O. Radium-223 in prostate cancer. N Engl J Med. 2013; 369:1659-1660.

18. Sweeney C, Chen YH, Carducci MA, et al. Impact on overall survival (OS) with chemohormonal therapy versus hormonal therapy for hormone-sensitive newly metastatic prostate cancer ( $\mathrm{mPrCa})$ : an ECOG-led phase III randomized trial. J Clin Oncol. 2014;32(5 suppl):abstract LBA2.
19. McDevitt MR, Ma D, Simon J, Frank RK, Scheinberg DA. Design and synthesis of ${ }^{225}$ Ac radioimmunopharmaceuticals. Appl Radiat Isot. 2002;57:841-847.

20. Mano H, Ishikawa F, Hirai H, Takaku F. Mutations of N-ras oncogene in myelodysplastic syndromes and leukemias detected by polymerase chain reaction. Jpn J Cancer Res. 1989;80:102-106.

21. Abou DS, Ku T, Smith-Jones PM. In vivo biodistribution and accumulation of ${ }^{89} \mathrm{Zr}$ in mice. Nucl Med Biol. 2011;38:675-681.

22. Vaziri B, Wu H, Dhawan AP, et al; SNMMI MIRD Committee. MIRD pamphlet no. 25: MIRDcell V2.0 software tool for dosimetric analysis of biologic response of multicellular populations. J Nucl Med. 2014;55:1557-1564.

23. Kreissl MC, Hanscheid H, Lohr M, et al. Combination of peptide receptor radionuclide therapy with fractionated external beam radiotherapy for treatment of advanced symptomatic meningioma. Radiat Oncol. 2012;7:99.

24. Ho AL, Grewal RK, Leboeuf R, et al. Selumetinib-enhanced radioiodine uptake in advanced thyroid cancer. $N$ Engl J Med. 2013;368:623-632.

25. Cheal SM, Xu H, Guo HF, et al. Preclinical investigation of multistep targeting of diaosialoganglioside GD2 using an IgG-scFv bispecific antibody with high affinity for GD2 and DOTA metal complex. Mol Cancer Ther. 2014;13:1803-1812.

26. Investigational new drug (IND) application. Food and Drug Administration website. http://www.fda.gov/Drugs/DevelopmentApprovalProcess/ HowDrugsareDevelopedandApproved/ApprovalApplications/InvestigationalNewDrugINDApplication/default.htm. Updated October 27, 2014. Accessed May 20, 2015.

27. Porter AT, McEwan AJ, Powe JE, et al. Results of a randomized phase-III trial to evaluate the efficacy of strontium- 89 adjuvant to local field ternal beam irradiation in the management of endocrine resistant metastatic prostate cancer. Int J Radiat Oncol Biol Phys. 1993;25:805-813.

28. McLendon RE, Archer GE, Garg PK, et al. Radiotoxicity of systematically administered $\left[{ }^{211} \mathrm{At}\right]$ astatide in $\mathrm{B} 6 \mathrm{C} 3 \mathrm{~F} 1$ and $\mathrm{BALB} / \mathrm{c}(\mathrm{nu} / \mathrm{nu})$ mice: a long-term survival study with histologic analysis. Int J Radiat Oncol Biol Phys. 1996; 35:69-80.

29. Kosterink JG, de Jonge MW, Smit EF, et al. Pharmacokinetics and scintigraphy of indium-111-DTPA-MOC-31 in small cell lung carcinoma. J Nucl Med. 1995;36:2356-2362.

30. Edelman MJ, Clamon G, Kahn D, Magram M, Lister-James J, Line BR. Targeted radiopharmaceutical therapy for advanced lung cancer: phase I trial of rhenium Re188 P2045, a somatostatin analog. J Thorac Oncol. 2009;4:1550-1554. 